\title{
Relationship between vitamin D in obstructive sleep apnea syndrome and psoriasis patients
}

\author{
Hülya Albayrak ${ }^{1}$ Nevin Fazlığlu², Bahadır Batar ${ }^{3}$, Mehmet Emin Yanık ${ }^{4}$ Mustafa Oran ${ }^{5}$, Nejat Altıntaş²
}

1Department of Dermatology, Namık Kemal University, Faculty of Medicine, Tekirdag, Turkey
2Department of Chest Diseases, Namık Kemal University, Faculty of Medicine, Tekirdag, Turkey
${ }^{3}$ Department of Medical Genetics, Namık Kemal University, Faculty of Medicine, Tekirdag, Turkey
${ }^{4}$ Department of Dermatology, Sancaktepe Region Hospital, Istanbul, Turkey
${ }^{5}$ Department of Internal Medicine, Namık Kemal University, Faculty of Medicine, Tekirdag, Turkey

Adv Dermatol Allergol 2022; XXXIX (2): 375-383

DOI: https://doi.org/10.5114/ada.2021.106031

\begin{abstract}
Introduction: Although psoriasis and obstructive sleep apnea syndrome (OSAS) are associated with systemic inflammation, studies on their potential bilateral relationship are not sufficient.

Aim: To investigate vitamin D levels and receptor gene polymorphisms in patients with OSAS and psoriasis and the associations with these diseases.

Material and methods: One hundred thirty-seven patients included in the study consisted of 4 different groups: group 1, those with both diseases; group 2, those with OSAS only; group 3, patients with psoriasis only; and group 4, healthy controls. The patients' serum calcium, phosphorus, AHI, Epworth Sleepiness Scale, Psoriasis Area Severity Index, and VDR Tagl, Apal, Bsml polymorphisms were compared.

Results: Vitamin D levels of groups 1, 2 and 3 were found to be lower than in controls. There was no statistically significant correlation between VDR Tagl, Apal, Bsml gene polymorphisms of the groups. Vitamin D levels were significantly higher in patients with heterozygous Apal genotype $(A / C)$ compared to patients with normal $(A / A)$ or homozygous mutant $(C / C)$ genotype $(p<0.05)$. No relationship was determined between VDR Tagl, Apal, Bsml, and the other parameters.

Conclusions: In our study, 1,25(OH) $)_{2}$-vitamin $\mathrm{D}_{3}$ levels were significantly lower in all disease groups compared to the control group. Although there is no difference between the groups in terms of VDR gene polymorphism, we think that there may be a bidirectional relationship between these diseases based on the low vitamin $D$ levels.
\end{abstract}

Key words: psoriasis, obstructive sleep apnea syndrome, vitamin D, VDR polymorphism.

\section{Introduction}

Psoriasis is a chronic, inflammatory disease characterized by nummular, scaly plaques on an erythematous background and its incidence varies from 1.5\% to 5.0\% [1]. Although it was only seen as a skin disease a few decades ago, it is now recognized as multi-system disease [2]. Seventy-three percent of psoriasis patients have at least one comorbidity such as arthritis, cardiovascular events, diabetes mellitus (DM), obesity, hypertension $(\mathrm{HT})$, or dyslipidaemia [3]. Autoimmune, genetic, and environmental factors are effective in the pathogenesis of psoriasis [4].

Obstructive sleep apnea syndrome (OSAS) is a disorder that causes intermittent hypoxemia and sleep disturbance due to partial or complete obstruction of the upper respiratory tract that recurs during sleep [5]. It affects approximately $2-4 \%$ of the society and has been observed twice as often in men than in women [6]. The risk of diabetes, pulmonary hypertension, coronary artery disease, heart failure, hypertension and stroke are increased in patients with OSAS [5].

As in psoriasis, inflammatory and immune imbalance has been described as major complications in the pathogenesis of OSAS [7, 8]. The relationship between psoriasis and OSAS has been studied in many studies, but the results vary and are limited [6, 9-12]. However, psoriasis is 4 times more common in OSAS patients than in the general population [13]. Psoriasis patients with OSAS in a study from Turkey were found to have an incidence of $13.7 \%$, which is considerably higher than the prevalence

Address for correspondence: Hülya Albayrak, Department of Dermatology, Namık Kemal University, Faculty of Medicine, Tekirdag, 59030, Turkey, phone: +90 50671550 65, e-mail: halbayrak@nku.edu.tr Received: 8.02.2021, accepted: 12.03.2021. 
in the general population [14]. Ilbay et al. [15] found this association at a rate of $61.4 \%$, while Karaca et al. [6] found it as $54.5 \%$.

Vitamin D is a fat-soluble vitamin and is known for its role in bone. In addition, vitamin D impacts many biological activities such as cellular proliferation and differentiation, immune system modulation, and muscle strengthening. Several studies have shown that serum levels of $1,25(\mathrm{OH})_{2}$-vitamin $\mathrm{D}_{3}\left(1,25(\mathrm{OH})_{2} \mathrm{D}_{3}\right)$ are significantly lower in patients with psoriasis compared with healthy controls $[16,17]$. Therapies based on vitamin D and its analogues have been used in psoriasis for decades and the deficiency of the active form of vitamin $D$ is assumed to play a role in the onset of psoriatic plaques $[18,19]$.

The vitamin D receptor (VDR) protein is a member of the nuclear receptor family of ligand-activated transcription factors [20]. The VDR gene encoding the VDR protein is located on the $12^{\text {th }}$ chromosome. VDR protein plays a role in important cellular biological events such as calcium (Ca) and phosphorus (P) haemostasis, cell differentiation, and apoptosis. The distribution of the vitamin D receptor in many tissues may explain the beneficial effects of vitamin D and its positive effects on the immune system at the molecular level. Apal, Bsml, Fokl, and Taql are the most common VDR gene polymorphisms, and studies investigating the effectiveness of these polymorphisms in psoriasis patients are conflicting [21].

\section{Aim}

In this study, we aimed to investigate the possible role of the most common Taql, Apal, and BsmI VDR gene polymorphisms in the susceptibility to psoriasis and OSAS disease.

\section{Material and methods}

Approval was obtained from the local ethics com mittee of Namık Kemal University for this research (No. 2018/97/07/03), which was conducted with the support of the Scientific Research Projects Coordination Unit (NKUBAP.02.GA.18.188). The Polysomnography (PSG) test was performed on patients with clinical suspicion of OSAS who applied to the Chest Diseases Clinic from November 2018 to November 2019. Based on the American Academy of Sleep Medicine (AASM) guidelines, a total of 137 patients were included in the study. Seventy eight were diagnosed with mild, moderate, and severe OSAS (apnea-hypopnea index $(\mathrm{AHI})>5$ ) and 59 were included without OSAS $(\mathrm{AHI}<5)$. Patients receiving systemic vitamin D treatment were excluded from the study. All patients included in the study were referred to the dermatology outpatient clinic and were examined for psoriasis. Demographic characteristics, PSG tests, and Epworth Sleepiness Scale (ESS) of the patients were recorded. The AHI score and Psoriasis Area and Severity Index (PASI) score of the psoriasis patients were calculated. Patients were classified into four groups in the study: group 1, those with both diseases $(n=33)$; group 2, those with OSAS only $(n=45)$; group 3 , patients with psoriasis only $(n=34)$; and group 4, healthy controls $(n=25)$.

\section{Blood samples and DNA extraction}

Two millilitres of venous blood samples were drawn from patients with psoriasis and OSAS as well as healthy individuals into EDTA tubes. Genomic DNA was isolated from peripheral blood mononuclear cells (PBMCs) using a DNA extraction kit (High Pure PCR Template Preparation Kit, Roche) according to the manufacturer's instructions. Genomic DNA was analysed for purity and concentration and then stored at $-20^{\circ} \mathrm{C}$ until use.

The human 1.25-dihydroxyvitamin $\mathrm{D}_{3}$ Enzyme-Linked Immunosorbent Assay (ELISA) kit (cat no. EA0057Hu, Bioassay Technology Laboratories Shanghai-China) was used to determine the $1,25(\mathrm{OH})_{2} \mathrm{D}_{3}$ levels. DVD/DHVD3 standards or samples were added to wells pre-coated with a monoclonal antibody. Then, a target antigen conjugated with biotin was added to the wells. After incubation, the values were read with the Biotek Elx-800 Microplate reader. This test detected $1,25(\mathrm{OH})_{2} \mathrm{D}_{3}$ with a specificity of $99 \%$. Sensitivity was evaluated according to guidelines from the Clinical \& Laboratory Standards Institute, Wayne, PA, USA. The sensitivity limit was $16.27 \mathrm{fmol} / \mathrm{l}$

\section{Genotyping}

Genotype distributions of vitamin D receptor gene rs731236 (Taql), rs7975232 (Apal), and rs1544410 (Bsml) single nucleotide polymorphisms (SNPs) were identified by Real-Time polymerase chain reaction (Real-Time P(R) technique. Genotyping was obtained using the LightCycler 2 (Roche Applied Science) instrument. Each Real-Time PCR was performed in a total volume of $20 \mu \mathrm{l}$ reaction mixture containing a $50 \mathrm{ng}$ final concentration of DNA, $1.6 \mu \mathrm{MgCl}_{2}(25 \mathrm{mM}), 1 \mu \mathrm{l}$ LightSNiP reagent mix (SimpleProbe, LightSNiP assays, TIB MolBiol, Berlin, Germany), 2 ul LightCycler ${ }^{\oplus}$ FastStart DNA Master HybProbe (Roche Diagnostics GmbH, Mannheim, Germany), and a variable amount of $\mathrm{H}_{2} \mathrm{O}$. Real-Time $\mathrm{PCR}$ reaction program consisted of the following cycle conditions: initial denaturation at $95^{\circ} \mathrm{C}$ for $10 \mathrm{~min}, 45$ cycles of denaturation at $95^{\circ} \mathrm{C}$ for $10 \mathrm{~s}$, annealing at $60^{\circ} \mathrm{C}$ for $10 \mathrm{~s}$, and extension at $72^{\circ} \mathrm{C}$ for $15 \mathrm{~s}$. The melting curve analysis of the amplification products was performed at $95^{\circ} \mathrm{C}$ for $20 \mathrm{~s}$, at $40^{\circ} \mathrm{C}$ for $20 \mathrm{~s}$, and at $85^{\circ} \mathrm{C}$ with a ramp rate of $0.2^{\circ} \mathrm{C} / \mathrm{s}$.

\section{Statistical analysis}

The Q-Q Plots and Histogram and Shapiro-Wilks test were used to test the normal distribution of the findings, and the Levene test was used to evaluate the homogeneity of the variables. A one-way ANOVA and Kruskal- 
Wallis test were used to compare the difference between groups. A Pearson $\chi^{2}$ test was used to compare qualitative variables for $\mathrm{H}$ test quantitative variables. Tukey and Siegel-Castellan tests were used in multiple comparisons. Statistical analysis was performed with Turcosa software (Turcosa Analytics, Turkey). A p-value of less than 0.05 was considered statistically significant.

\section{Results}

The demographic characteristics of the patients and healthy controls participating in the study are given in Table 1. Vitamin D levels of groups 1, 2, and 3 were significantly lower than the healthy control group $4(p=0.019)$. Vitamin D levels of groups 1, 2, and 3 were similar. The highest ESS level was observed in group 2. The ESS level in group 2 was statistically higher than in groups 1 and 4 . Body mass index (BMI) was higher in group 2 compared with other groups, and the difference with group 3 was statistically significant. The average age of group 1 and group 2 was significantly higher than in group 3 and group 4.

The VDR gene polymorphism distribution of the groups is given in Table 2. When VDR Tagl, VDR Apal, and VDR Bsml gene polymorphisms were compared, no statistically significant relationship was observed between the groups $(p>0.05)$.

A statistically significant difference was found in the comparison of the VDR Apal gene polymorphism genotype distributions (homozygous, heterozygous, and mutant) and the medians of the vitamin D variable $(p=0.019)$. However, no significant difference was found in the comparison of AHI, ESS, PASI, age, Ca, P, Albumin, and $\mathrm{BMI}$ variables $(p>0.05)$. According to the multiple comparison test, vitamin D levels were found significantly higher in patients with Apal heterozygous genotype $(A / C)$ compared to patients with normal (A/A) or homozygous mutant $(C / C)$ genotype $(p<0.05)$. Although vitamin D was significantly higher than in the other two groups in terms of this heterozygous genotype, the disease severity scores $\mathrm{AHI}$ and PASI were found to be higher (Table 3).

No significant difference was found in the comparison of VDR Bsml gene polymorphism, genotype distributions, and vitamin D, AHI, ESS, PASI, age, Ca, P, Albumin, and $\mathrm{BMI}$ variables $(p>0.05)$ (Table 4$)$.

There was no significant difference in the comparison of VDR Tagl gene polymorphism genotype distributions and vitamin $\mathrm{D}, \mathrm{AHI}$, ESS, PASI, age, $\mathrm{Ca}, \mathrm{P}$, and $\mathrm{BMI}$ variables $(p>0.05)$. However, the albumin value of the mutant group was higher than in the other groups $(p=0.015)$ (Table 5).

When the genotype distributions of VDR Tag1, VDR Apal, and VDR Bsml gene polymorphisms were compared with the presence or absence of OSAS and the presence or absence of psoriasis; no significant correlation was found $(p>0.05)$ (Table 6).

\section{Discussion}

In this study, we investigated the link between patients with psoriasis and OSAS and the role of the VDR gene polymorphisms. We determined that vitamin D was lower in both psoriasis and OSAS patients than

Table 1. Demographic characteristics of the patients participating in the study

\begin{tabular}{|c|c|c|c|c|c|}
\hline Parameter & Group 1 & Group 2 & Group 3 & Group 4 & $P$-value \\
\hline Vitamin D & $n=28$ & $n=43$ & $n=27$ & $n=20$ & 0.019 \\
\hline$[\mathrm{fmol} / \mathrm{l}]$ & 1652.5 & 2010.8 & 1795.5 & 6133.7 & \\
\hline$($ mean \pm SD) & $(989.9-3857.4)^{a}$ & $(1031.7-5621.9)^{a}$ & $(1069.9-5003.2)^{a}$ & $(2902.3-7878.3)^{\mathrm{b}}$ & \\
\hline ESS median & $n=26$ & $n=43$ & $n=27$ & $n=20$ & 0.023 \\
\hline (Q25-Q75) & $3.0(2.0-6.25)^{a}$ & $6.0(3.0-11.0)^{b}$ & $4.0(3.0-6.0)^{\mathrm{ab}}$ & $3.0(1.25-4.0)^{\mathrm{a}}$ & \\
\hline Age [years] & $n=28$ & $n=43$ & $n=27$ & $n=20$ & $<0.001$ \\
\hline$($ mean $\pm S D)$ & $56.3 \pm 11.3^{a}$ & $53.6 \pm 12.4^{a}$ & $40.8 \pm 14.5^{b}$ & $41.9 \pm 10.56^{b}$ & \\
\hline $\mathrm{Ca}[\mathrm{mg} / \mathrm{dl}]$ & $n=27$ & $n=36$ & $n=27$ & $n=19$ & 0.104 \\
\hline$($ mean \pm SD) & $9.8 \pm 0.4$ & $9.6 \pm 0.4$ & $9.7 \pm 0.4$ & $9.6 \pm 0.4$ & \\
\hline$P[\mathrm{mg} / \mathrm{dl}]$ & $n=26$ & $n=33$ & $n=25$ & $n=19$ & 0.680 \\
\hline$($ mean $\pm S D)$ & $3.35 \pm 0.67$ & $3.38 \pm 0.79$ & $3.46 \pm 0.56$ & $3.22 \pm 0.48$ & \\
\hline PTH [pg/ml] & $n=26$ & $n=30$ & $n=25$ & $n=18$ & 0.043 \\
\hline$($ mean $\pm S D)$ & $56.89 \pm 24.42^{\mathrm{ab}}$ & $66.66 \pm 27.22^{a}$ & $53.34 \pm 15.53^{\mathrm{ab}}$ & $48.53 \pm 20.72^{a}$ & \\
\hline Albumin [g/dl] & $n=27$ & $n=38$ & $n=26$ & $n=19$ & 0.191 \\
\hline$($ mean \pm SD) & $4.61 \pm 0.21$ & $4.55 \pm 0.35$ & $4.41 \pm 0.31$ & $4.41 \pm 0.31$ & \\
\hline BMI & $n=26$ & $n=43$ & $n=27$ & $n=20$ & $<0.001$ \\
\hline$($ mean $\pm S D)$ & $30.48 \pm 4.32^{\mathrm{ac}}$ & $32.75 \pm 4.98^{a}$ & $26.63 \pm 3.30^{b}$ & $29.29 \pm 5.00^{\text {bc }}$ & \\
\hline
\end{tabular}

BMI - body mass index, Ca - calcium, ESS - Epworth Sleepiness Scale, PTH - parathyroid hormone, P - phosphorus. Data are expressed as median (25-75 percentile) or arithmetic mean \pm standard deviation $(\bar{x} \pm s)$. According to the post hoc test (TUKEY or Siegel-Castellan), the difference between the groups is statistically significant in cases with different alphabetic superscripts. 
Table 2. Distribution of VDR gene polymorphisms by groups

\begin{tabular}{|c|c|c|c|c|c|}
\hline \multirow[t]{2}{*}{ Polymorphisms } & \multicolumn{4}{|c|}{ Groups } & \multirow[t]{2}{*}{$P$-value } \\
\hline & $1, n(\%)$ & $2, n(\%)$ & $3, n(\%)$ & $4, n(\%)$ & \\
\hline \multicolumn{5}{|l|}{ VDR Tagl: } & 0.924 \\
\hline Homozygote (TT) & $10(35.7)$ & $14(32.6)$ & $12(44.4)$ & $8(40.0)$ & \\
\hline Heterozygote (TC) & $13(46.4)$ & $21(48.8)$ & $10(37.0)$ & $10(50.0)$ & \\
\hline Mutant (CC) & $5(17.9)$ & $8(18.6)$ & $5(18.5)$ & $2(10.0)$ & \\
\hline \multicolumn{5}{|l|}{ VDR Apal: } & 0.787 \\
\hline Homozygote (CC) & $6(21.4)$ & $11(2.6)$ & $6(22.2)$ & $5(25.0)$ & \\
\hline Heterozygote (CA) & $11(39.3)$ & $20(46.5)$ & $9(33.3)$ & $10(50.0)$ & \\
\hline Mutant (AA) & $11(39.3)$ & $12(27.9)$ & $12(44.4)$ & $5(25.0)$ & \\
\hline \multicolumn{5}{|l|}{ VDR Bsml: } & 0.888 \\
\hline Homozygote (GG) & $9(32.1)$ & $15(34.9)$ & $12(44.4)$ & $6(30.0)$ & \\
\hline Heterozygote (GA) & $13(46.4)$ & $18(41.9)$ & $10(37.0)$ & $11(55.0)$ & \\
\hline Mutant (AA) & $6(21.4)$ & $10(23.3)$ & $5(18.5)$ & $3(15.0)$ & \\
\hline
\end{tabular}

Table 3. Relationships between Apal gene polymorphism genotype distribution and clinical characteristics of patients

\begin{tabular}{|c|c|c|c|c|}
\hline \multirow[t]{2}{*}{ Variables } & \multicolumn{3}{|c|}{ VDR Apal (rs7975232) } & \multirow[t]{2}{*}{$P$-value } \\
\hline & Homozygote & Heterozygote & Mutant & \\
\hline $\begin{array}{l}\text { Vitamin D [fmol/l] } \\
\text { median (Q25-Q75) }\end{array}$ & $\begin{array}{c}n=28 \\
1833.5 \\
(836.5-3110.7)^{a}\end{array}$ & $\begin{array}{c}n=50 \\
3852.6 \\
(1365.5-8282.7)^{b}\end{array}$ & $\begin{array}{c}n=40 \\
1804 \\
(1203.0-5162.5)^{a}\end{array}$ & 0.019 \\
\hline $\begin{array}{l}\mathrm{AHI} \\
\text { median (Q25-Q75) }\end{array}$ & $\begin{array}{c}n=28 \\
23.0(1.23-45.18)\end{array}$ & $\begin{array}{c}n=50 \\
31.85(1.5-58.85)\end{array}$ & $\begin{array}{c}n=40 \\
18.10(1.68-40.90)\end{array}$ & 0.555 \\
\hline $\begin{array}{l}\text { ESS } \\
\text { median (Q25-Q75) }\end{array}$ & $\begin{array}{c}n=28 \\
4.0(2.0-7.0)\end{array}$ & $\begin{array}{c}n=49 \\
4.0(2.0-6.5)\end{array}$ & $\begin{array}{c}n=39 \\
4.0(2.0-8.0)\end{array}$ & 0.600 \\
\hline $\begin{array}{l}\text { PASI } \\
\text { median (Q25-Q75) }\end{array}$ & $\begin{array}{c}n=12 \\
4.90(2.35-7.65)\end{array}$ & $\begin{array}{c}n=16 \\
5.50(0.38-8.30)\end{array}$ & $\begin{array}{c}n=21 \\
1.60(0.50-5.45)\end{array}$ & 0.194 \\
\hline $\begin{array}{l}\text { Age [years] } \\
(\text { mean } \pm S D)\end{array}$ & $\begin{array}{c}n=28 \\
48.82 \pm 16.54\end{array}$ & $\begin{array}{c}n=50 \\
49.74 \pm 12.74\end{array}$ & $\begin{array}{c}n=40 \\
49.20 \pm 13.79\end{array}$ & 0.960 \\
\hline $\begin{array}{l}\mathrm{Ca}[\mathrm{mg} / \mathrm{dl}] \\
(\mathrm{mean} \pm \mathrm{SD})\end{array}$ & $\begin{array}{c}n=26 \\
9.70 \pm 0.43\end{array}$ & $\begin{array}{c}n=46 \\
9.67 \pm 0.40\end{array}$ & $\begin{array}{c}n=37 \\
9.74 \pm 0.38\end{array}$ & 0.696 \\
\hline $\begin{array}{l}\mathrm{P}[\mathrm{mg} / \mathrm{dl}] \\
(\text { mean } \pm \mathrm{SD})\end{array}$ & $\begin{array}{c}n=26 \\
3.83 \pm 2.67\end{array}$ & $\begin{array}{c}n=43 \\
3.32 \pm 0.59\end{array}$ & $\begin{array}{c}n=34 \\
3.36 \pm 0.56\end{array}$ & 0.749 \\
\hline $\begin{array}{l}\text { PTH }[\mathrm{pg} / \mathrm{ml}] \\
(\mathrm{mean} \pm \mathrm{SD})\end{array}$ & $\begin{array}{c}n=25 \\
62.00 \pm 26.30\end{array}$ & $\begin{array}{c}n=42 \\
57.57 \pm 24.97\end{array}$ & $\begin{array}{c}n=32 \\
53.68 \pm 18.64\end{array}$ & 0.417 \\
\hline $\begin{array}{l}\text { Albumin }[\mathrm{g} / \mathrm{dl}] \\
(\text { mean } \pm \mathrm{SD})\end{array}$ & $\begin{array}{c}n=27 \\
4.59 \pm 0.26\end{array}$ & $\begin{array}{c}n=48 \\
4.47 \pm 0.32\end{array}$ & $\begin{array}{c}n=35 \\
4.62 \pm 0.30\end{array}$ & 0.054 \\
\hline $\begin{array}{l}\text { BMI } \\
(\text { mean } \pm \text { SD) }\end{array}$ & $\begin{array}{c}n=28 \\
30.81 \pm 6.02\end{array}$ & $\begin{array}{c}n=49 \\
30.30 \pm 4.94\end{array}$ & $\begin{array}{c}n=39 \\
29.69 \pm 4.39\end{array}$ & 0.659 \\
\hline
\end{tabular}

AHI-Apnea-Hypopnea Index, BMI - body mass index, Ca-calcium, ESS - Epworth Sleepiness Scale, PASI - Psoriasis Area and Severity Index, PTH - parathyroid hormone, $P$ - phosphorus. Data are expressed as median (25-75 percentile) or arithmetic mean \pm standard deviation ( $\bar{x} \pm s)$. According to the post hoc test (TUKEY or Siegel-Castellan), the difference between the groups is statistically significant in cases with different alphabetic superscripts.

in healthy controls. However, we did not observe any relationship between VDR gene polymorphism and these diseases. BMI was higher in patients with OSAS than in other groups, and the difference with psoriasis patients was statistically significant. The average age of group 1 and group 2 was significantly higher than in group 3 and group 4. This situation was thought to be related to the fact that OSAS has been documented as 
Table 4. Relationships between Bsml gene polymorphism genotype distribution and clinical characteristics of patients

\begin{tabular}{|c|c|c|c|c|}
\hline \multirow[t]{2}{*}{ Variables } & \multicolumn{3}{|c|}{ VDR Bsml (rs1544410) } & \multirow[t]{2}{*}{$P$-value } \\
\hline & Homozygote & Heterozygote & Mutant & \\
\hline $\begin{array}{l}\text { Vitamin } D \\
{[\mathrm{fmol} / \mathrm{l}]} \\
\text { median (Q25-Q75) }\end{array}$ & $\begin{array}{c}n=42 \\
2225.5 \\
(1004.8-6466.1)\end{array}$ & $\begin{array}{c}n=52 \\
3721.3 \\
(1326.5-7156.5)\end{array}$ & $\begin{array}{c}n=24 \\
1804 \\
(1009.8-2977.8)\end{array}$ & 0.178 \\
\hline $\begin{array}{l}\mathrm{AHI} \\
\text { median (Q25-Q75) }\end{array}$ & $\begin{array}{c}n=42 \\
21.1(1.4-46.0)\end{array}$ & $\begin{array}{c}n=52 \\
20.3(1.3-51.4)\end{array}$ & $\begin{array}{c}n=24 \\
21.9(2.2-48.5)\end{array}$ & 0.775 \\
\hline $\begin{array}{l}\text { ESS } \\
\text { median (Q25-Q75) }\end{array}$ & $\begin{array}{c}n=42 \\
4(2.8-7.0)\end{array}$ & $\begin{array}{c}n=50 \\
4(2.0-7.0)\end{array}$ & $\begin{array}{c}n=24 \\
5(2.0-9.0)\end{array}$ & 0.831 \\
\hline $\begin{array}{l}\text { PASI } \\
\text { median (Q25-Q75) }\end{array}$ & $\begin{array}{c}n=18 \\
5.50(3.50-8.05)\end{array}$ & $\begin{array}{c}n=22 \\
1.85(0.00-7.13)\end{array}$ & $\begin{array}{c}n=9 \\
1.40(1.00-6.70)\end{array}$ & 0.100 \\
\hline $\begin{array}{l}\text { Age [years] } \\
(\text { mean } \pm \text { SD) }\end{array}$ & $\begin{array}{c}n=42 \\
47.14 \pm 16.19\end{array}$ & $\begin{array}{c}n=52 \\
51.63 \pm 11.67\end{array}$ & $\begin{array}{c}n=24 \\
48.21 \pm 14.10\end{array}$ & 0.274 \\
\hline $\begin{array}{l}\mathrm{Ca}[\mathrm{mg} / \mathrm{dl}] \\
(\text { mean } \pm \mathrm{SD})\end{array}$ & $\begin{array}{c}n=40 \\
9.68 \pm 0.41\end{array}$ & $\begin{array}{c}n=47 \\
9.69 \pm 0.39\end{array}$ & $\begin{array}{c}n=22 \\
9.78 \pm 0.39\end{array}$ & 0.614 \\
\hline $\begin{array}{l}\mathrm{P}[\mathrm{mg} / \mathrm{dl}] \\
(\text { mean } \pm \mathrm{SD})\end{array}$ & $\begin{array}{c}n=38 \\
3.42 \pm 0.82\end{array}$ & $\begin{array}{c}n=44 \\
3.30 \pm 0.52\end{array}$ & $\begin{array}{c}n=21 \\
3.38 \pm 0.57\end{array}$ & 0.699 \\
\hline $\begin{array}{l}\text { PTH }[\mathrm{pg} / \mathrm{ml}] \\
(\text { mean } \pm \text { SD) }\end{array}$ & $\begin{array}{c}n=37 \\
59.14 \pm 25.33\end{array}$ & $\begin{array}{c}n=42 \\
59.45 \pm 23.70\end{array}$ & $\begin{array}{c}n=20 \\
50.05 \pm 18.36\end{array}$ & 0.291 \\
\hline $\begin{array}{l}\text { Albumin [g/dl] } \\
\text { (mean } \pm \text { SD) }\end{array}$ & $\begin{array}{c}n=40 \\
4.56 \pm 0.31\end{array}$ & $\begin{array}{c}n=49 \\
4.49 \pm 0.29\end{array}$ & $\begin{array}{c}n=21 \\
4.65 \pm 0.32\end{array}$ & 0.173 \\
\hline $\begin{array}{l}\mathrm{BMI} \\
(\text { mean } \pm \mathrm{SD})\end{array}$ & $\begin{array}{c}n=42 \\
30.41 \pm 5.52\end{array}$ & $\begin{array}{c}n=50 \\
30.13 \pm 4.63\end{array}$ & $\begin{array}{c}n=24 \\
30.07 \pm 5.14\end{array}$ & 0.952 \\
\hline
\end{tabular}

AHI-Apnea-Hypopnea Index, BMI - body mass index, Ca-calcium, ESS - Epworth Sleepiness Scale, PASI - Psoriasis Area and Severity Index, PTH - parathyroid hormone, $P$ - phosphorus. Data are expressed as median (25-75 percentile) or arithmetic mean \pm standard deviation $(\bar{x} \pm S)$. According to the post hoc test (TUKEY or Siegel-Castellan), the difference between the groups is statistically significant in cases with different alphabetic superscripts.

Table 5. Relationship between Tagl gene polymorphism genotype distribution and clinical characteristics of patients

\begin{tabular}{|c|c|c|c|c|}
\hline \multirow[t]{2}{*}{ Variables } & \multicolumn{3}{|c|}{ VDR Tagl (rs731236) } & \multirow[t]{2}{*}{$P$-value } \\
\hline & Homozygote & Heterozygote & Mutant & \\
\hline $\begin{array}{l}\text { Vitamin D } \\
{[\mathrm{fmol} / \mathrm{l}]} \\
\text { median (Q25-Q75) }\end{array}$ & $\begin{array}{c}n=44 \\
2329.7 \\
(1021.8-6908.2)\end{array}$ & $\begin{array}{c}n=54 \\
3721.3 \\
(1358.4-7027.6)\end{array}$ & $\begin{array}{c}n=20 \\
1715 \\
(970.4-2279.9)\end{array}$ & 0.105 \\
\hline $\begin{array}{l}\text { AHI } \\
\text { median (Q25-Q75) }\end{array}$ & $\begin{array}{c}n=44 \\
19.7(1.3-46.1)\end{array}$ & $\begin{array}{c}n=54 \\
20.3(1.6-51.3)\end{array}$ & $\begin{array}{c}n=20 \\
30.0(1.9-53.4)\end{array}$ & 0.584 \\
\hline $\begin{array}{l}\text { ESS } \\
\text { median (Q25-Q75) }\end{array}$ & $\begin{array}{c}n=44 \\
4(2.3-7.0)\end{array}$ & $\begin{array}{c}n=52 \\
4.0(2.2-7.0)\end{array}$ & $\begin{array}{c}n=2 \\
3.5(2.0-8.0)\end{array}$ & 0.864 \\
\hline $\begin{array}{l}\text { PASI } \\
\text { median (Q25-Q75) }\end{array}$ & $\begin{array}{c}n=19 \\
5.00(2.00-6.70)\end{array}$ & $\begin{array}{c}n=22 \\
6.13(0.00-8.50)\end{array}$ & $\begin{array}{c}n=8 \\
1.30(1.00-6.95)\end{array}$ & 0.273 \\
\hline $\begin{array}{l}\text { Age [years] } \\
(\text { mean } \pm \text { SD) }\end{array}$ & $\begin{array}{c}n=44 \\
46.18 \pm 15.43\end{array}$ & $\begin{array}{c}n=54 \\
52.26 \pm 11.94\end{array}$ & $\begin{array}{c}n=20 \\
48.4 \pm 14.61\end{array}$ & 0.094 \\
\hline $\begin{array}{l}\mathrm{Ca}[\mathrm{mg} / \mathrm{dl}] \\
(\text { mean } \pm \mathrm{SD})\end{array}$ & $\begin{array}{c}n=42 \\
9.72 \pm 0.36\end{array}$ & $\begin{array}{c}n=49 \\
9.66 \pm 0.42\end{array}$ & $\begin{array}{c}n=18 \\
9.77 \pm 0.41\end{array}$ & 0.550 \\
\hline $\begin{array}{l}\mathrm{P}[\mathrm{mg} / \mathrm{dl}] \\
(\text { mean } \pm \mathrm{SD})\end{array}$ & $\begin{array}{c}n=41 \\
3.41 \pm 0.82\end{array}$ & $\begin{array}{c}n=44 \\
3.29 \pm 0.52\end{array}$ & $\begin{array}{c}n=18 \\
3.45 \pm 0.54\end{array}$ & 0.563 \\
\hline $\begin{array}{l}\text { PTH [pg/ml] } \\
(\text { mean } \pm \text { SD) }\end{array}$ & $\begin{array}{c}n=40 \\
58.98 \pm 24.59\end{array}$ & $\begin{array}{c}n=42 \\
58.63 \pm 23.85\end{array}$ & $\begin{array}{c}n=17 \\
50.84 \pm 19.50\end{array}$ & 0.448 \\
\hline $\begin{array}{l}\text { Albumin [g/dl] } \\
\text { (mean } \pm \text { SD) }\end{array}$ & $\begin{aligned} n & =42 \\
4.57 & \pm 0.27^{\mathrm{ab}}\end{aligned}$ & $\begin{array}{c}n=51 \\
4.47 \pm 0.31^{a}\end{array}$ & $\begin{array}{c}n=17 \\
4.71 \pm 0.32^{b}\end{array}$ & 0.015 \\
\hline $\begin{array}{l}\mathrm{BMI} \\
(\text { mean } \pm \mathrm{SD})\end{array}$ & $\begin{array}{c}n=44 \\
30.68 \pm 5.7\end{array}$ & $\begin{array}{c}n=52 \\
29.94 \pm 4.36\end{array}$ & $\begin{array}{c}n=20 \\
29.93 \pm 5.03\end{array}$ & 0.747 \\
\hline
\end{tabular}

AHI-Apnea-Hypopnea Index, BMI - body mass index, Ca-calcium, ESS - Epworth Sleepiness Scale, PASI - Psoriasis Area and Severity Index, PTH - parathyroid hormone, $P$ - phosphorus. Data are expressed as median (25-75 percentile) or arithmetic mean \pm standard deviation ( $\bar{x} \pm s)$. According to the post hoc test (TUKEY or Siegel-Castellan), the difference between the groups is statistically significant in cases with different alphabetic superscripts. 
Table 6. Relationship between OSAS and psoriasis patients and VDR gene polymorphisms

\begin{tabular}{|c|c|c|c|c|c|c|}
\hline \multirow[t]{2}{*}{ Polymorphisms } & \multicolumn{2}{|c|}{ OSAS n (\%) } & \multirow[t]{2}{*}{$P$-value } & \multicolumn{2}{|c|}{ Psoriasis $n$ (\%) } & \multirow[t]{2}{*}{$P$-value } \\
\hline & $(-)$ & $(+)$ & & $(-)$ & $(+)$ & \\
\hline VDR Tagl: & & & 0.621 & & & 0.724 \\
\hline Homozygote & $20(42.6)$ & $24(33.8)$ & & $22(34.9)$ & $22(40.0)$ & \\
\hline Heterozygote & $20(42.6)$ & 34 (47.9) & & $31(49.2)$ & $23(41.8)$ & \\
\hline Mutant & $7(14.9)$ & $13(18.3)$ & & $10(15.9)$ & $10(18.2)$ & \\
\hline VDR Apal: & & & 0.908 & & & 0.230 \\
\hline Homozygote & $11(23.4)$ & 17 (23.9) & & $16(25.4)$ & $12(21.8)$ & \\
\hline Heterozygote & $19(40.4)$ & 31 (43.7) & & $30(47.6)$ & $20(36.4)$ & \\
\hline Mutant & 17 (36.2) & $23(32.4)$ & & $17(27.0)$ & $23(41.8)$ & \\
\hline VDR Bsml: & & & 0.745 & & & 0.853 \\
\hline Homozygote & $18(38.3)$ & $24(33.8)$ & & $21(33.3)$ & $21(38.2)$ & \\
\hline Heterozygote & $21(44.7)$ & 31 (43.7) & & $29(46.0)$ & $23(41.8)$ & \\
\hline Mutant & $8(17.0)$ & $16(22.5)$ & & 13 (20.6) & $11(20.0)$ & \\
\hline
\end{tabular}

a disease of advanced age, while psoriasis has been observed in younger ages. There was no significant difference in the group with both diseases compared to the group with only OSAS. This situation can be explained by the fact that OSAS is seen especially in older psoriasis patients.

Different results have been reported in the literature regarding the relationship between vitamin D levels and psoriasis patients. In a study by Gisondi et al., in which vitamin D levels were evaluated in patients with chronic plaque psoriasis, increased vitamin D deficiency was found, especially in winter months [16]. However, a causal relationship was not established between vitamin D deficiency and psoriasis. In a study evaluating psoriasis along with inflammatory skin diseases, a significantly lower vitamin D level was found in all patients with atopic dermatitis, infectious diseases, and psoriasis compared to the control group. The lowest vitamin D level was found in the patient group with psoriasis [22]. In another study, it was shown that the duration of psoriasis disease had an effect on vitamin D levels and lower levels were found in patients with psoriasis compared to the control group [23]. In a meta-analysis evaluating vitamin D levels in a total of 10 studies and 571 psoriasis patients, it was determined that vitamin D levels were low in psoriasis patients and a negative correlation was found between PASI score and vitamin D level [21]. In addition, they suggested that vitamin D may play a role in the psoriasis pathogenesis in this meta-analysis [21]. In a study from 2018, a positive correlation was found between vitamin D and psoriasis in 14 studies (1249 patients with psoriasis and 680 healthy controls), while no correlation was found between psoriasis and vitamin D in six studies [24]. In our study, we found that vitamin D levels in psoriasis patients were significantly lower than in the control group. This result is consistent with many previous studies [22, 23].

Studies showing the relationship between sleep disorders and low vitamin D levels have increased continuously in recent years. In addition to risk factors such as age, obesity, hypertension, kidney disease, and diabetes associated with OSAS, vitamin D deficiency is also considered as a risk factor [25]. Vitamin D deficiency can cause sleep disorders with increased inflammatory factors including tumour necrosis factor $\alpha$ (TNF- $\alpha$ ), interleukin (IL)-1, and prostaglandin D2 (PGD2). Vitamin D deficiency can increase the risk of OSA with adenotonsillar hypertrophy, airway muscle myopathy, and/or chronic rhinitis [26]. The study by Piovezan et al. [27] showed that there is a significant relationship between moderate and severe OSAS and low vitamin D levels. In a study by Mete et al. [28], vitamin D deficiency was observed in the OSAS group compared with the control group. In addition, a significant correlation was found between the severity of OSAS and vitamin D deficiency. In the study by Barcelo et al. [29], vitamin D levels in patients with severe OSAS were found to be lower than in patients with other OSASs, but they were not statistically significant. In the study by Erden et al. [30], OSAS was found to be associated with high Bisphenol A (BPA) and parathyroid hormone (PTH) levels and low vitamin D levels. In a meta-analysis evaluating vitamin D levels in OSAS patients, 14 studies were evaluated and vitamin D levels were found to be reduced in evaluation [5]. In our study, in accordance with this literature meta-analysis, and many other studies, we found that vitamin D levels were lower than the control group.

Gupta et al. [8] suggested that there was a bilateral relationship between OSAS and psoriasis in a study investigating psoriasis and other dermatological diseases in OSAS patients. In addition, the proinflammatory condition found in OSAS patients has been shown to exacer- 
bate dermatoses such as psoriasis [8]. In an observational study by Gabryelska et al. [13], psoriasis was observed to be more than 4 times higher in OSAS patients compared to normal individuals. Similarly, Papadavid et al. [31] reported that the incidence of psoriasis in OSAS patients was $9.5 \%$ compared to $2.9 \%$ in patients without OSAS.

As observed, vitamin D levels in patients with both OSAS and psoriasis are lower than in healthy controls. However, some studies have indicated that vitamin D may play a role in the pathogenesis of OSAS and psoriasis. At the same time, there are many studies investigating the association between OSAS and psoriasis. Our hypothesis for this study was that VDR gene polymorphisms may play a role in the relationship between OSAS and psoriasis. We did not find any relationship with VDR gene polymorphism and OSAS and psoriasis. In addition, when we evaluated these polymorphisms one by one, we did not find a significant difference for all patients and control groups. No relationship was found between VDR Bsml and Taql gene polymorphisms and the clinical features in the groups we examined. The Apal heterozygous genotype was found to be associated with significantly increased vitamin D levels; although PASI and AHI values, which are indicators of psoriasis and OSAS disease severity, were found to be high.

The study by Ragia et al. [32] is the first clinical genetic study investigating the effect of VDR polymorphism on OSAS. The relationship between VDR Fokl, Bsml, Apal, and Taql gene polymorphisms with serum vitamin D levels and OSAS was investigated. The frequency of Fokl CC genotype was found to be significantly higher in patients with OSAS compared to the control group. FokI CC phenotype was found to be significantly associated with low vitamin D levels in patients with OSAS according to $\mathrm{CT}$ and TT phenotypes. It has also been shown that the VDR FokI polymorphism is associated with excessive daytime sleepiness. Kirac et al. [33] investigated VDR Fokl (rs2228570) and Bsml (rs1544410) and vitamin D-binding protein rs4588 and rs7041 mutations in OSAS patients. They reported that CA genotype in rs4588, CC genotype in rs2228570, and AA genotype in rs1544410 mutations were statistically significant in patients. Interestingly, the TC genotype in rs2228570 and GA genotype in rs1544410 mutations were statistically significant in controls. When the relationship between risk factors and genotypes was examined, they found a statistically significant relationship in terms of BMI, waist circumference, AHI, ESS, vitamin $D$, and triglyceride levels. It has been shown in previous studies that VDR allelic variability affects VDR mRNA levels. The VDR gene is located on chromosome 12q13.11. and the Bsml and Apal gene polymorphisms occur in intron 8. The Taql gene polymorphism is defined in exon 9, and Apal and Taql polymorphisms have been shown to lead to impaired binding at the receptor level [21]. In our study, although vitamin D level was found to be significantly high in Apal, the fact that disease scores were also high can be explained by the attachment disorder caused by Apal. Statistically low levels of vitamin D in psoriasis and OSAS patient groups may increase the susceptibility to the disease and suggest that the VDR gene mutation that mediates the vitamin D effect may also be effective.

Different results of the relationship between VDR polymorphisms and susceptibility to psoriasis in different populations have been described. VDR Apal polymorphism has been reported to be associated with psoriasis in Korea [34, 35], China [36], and the Turkish population [37, 38], while that in Japan [39], Italy [40], Croatia [41] or Egypt [42] it has not been recognized as a risk factor for psoriasis cases. Li et al. [43] reported that VDR rs7975232 polymorphism did not have a significant effect on susceptibility to psoriasis in their meta-analysis evaluating the VDR rs7975232 polymorphism and psoriasis susceptibility of 13 case-control studies with 1,654 cases and 1,991 controls. In the same study, it was shown that VDR Bsml polymorphism was not associated with susceptibility to psoriasis in 13 studies involving 1,620 cases and 2,001 controls. Similarly, VDR Taql polymorphism was not shown to be associated with susceptibility to psoriasis in studies involving 1,690 cases and 1,857 controls. In two previous case-control studies conducted in the Turkish population, a relationship between VDR Apal polymorphism and psoriasis susceptibility was reported [37, 38]. However, we did not observe any predisposition among all three VDR gene polymorphisms in our study. Although our results contradict the results of Kaya [38] and Dayangac-Erden [37], our results are consistent with the meta-analysis results of Li et al. [43].

In a meta-analysis [21], the VDR gene mutations in Caucasians and Asians were not shown to be associated with psoriasis susceptibility; but Taql polymorphism, which again causes VDR binding imbalance, was associated with psoriasis in the Caucasian race. In our study, no statistical difference was observed in the VDR gene polymorphism between the groups. Contrary to the low vitamin $D$ levels seen in both diseases, both disease scores were found to be high at a level that did not reach statistical significance; although vitamin D levels were found to be significantly higher in the group with heterozygous Apal. It shows that this condition, which creates vitamin D attachment disorder, may contribute to the exacerbation of the disease. This may indicate that in addition to Taql, which has been shown in previous studies and creates an imbalance in binding to the receptor, Apal may also cause an exacerbation of the disease in the psoriasis and OSAS groups through vitamin D attachment disorder. The low number of patient groups may be the reason why Apal heterozygous polymorphism does not reach significance in psoriasis and OSAS.

\section{Conclusions}

Our study is the first study to evaluate the link and the common pathogenesis of psoriasis and OSAS togeth- 
er with VDR gene polymorphisms. We determined that VDR gene polymorphisms did not pose a risk for both diseases. However, more studies are needed to investigate the association between OSAS and psoriasis.

\section{Acknowledgments}

A this study was supported by Namık Kemal University Scientific Research Projects Coordination Unit (Project Number NKUBAP.02.GA.18.188).

The authors appreciate the technical assistance provided by the Namık Kemal University Scientific Research Projects Coordination Unit.

\section{Conflict of interest}

The authors declare no conflict of interest.

\section{References}

1. Gao Y, Sun W, Cha X, Wang H. 'Psoriasis 1' reduces T lymphocyte mediated inflammation in patients with psoriasis by inhibiting vitamin D receptor mediated STAT4 inactivation. Int J Mol Med 2020; 46: 1538-50.

2. Lotti T, Hercogova J, Prignano F. The concept of psoriatic disease: can cutaneous psoriasis any longer be separated by the systemic comorbidities? Dermatol Ther 2010; 23: 119-22.

3. Puig-Sanz L. Psoriasis, a systemic disease? Actas Dermosifiliogr 2007; 98: 396-402.

4. Zuccotti E, Oliveri M, Girometta C, et al. Nutritional strategies for psoriasis: current scientific evidence in clinical trials. Eur Rev Med Pharmacol Sci 2018; 22: 8537-51.

5. Neighbors CLP, Noller MW, Song SA, et al. Vitamin D and obstructive sleep apnea: a systematic review and metaanalysis. Sleep Med 2018; 43: 100-8.

6. Karaca S, Fidan F, Erkan F, et al. Might psoriasis be a risk factor for obstructive sleep apnea syndrome? Sleep Breath 2013; 17: 275-80.

7. Hirotsu C, Nogueira H, Albuquerque RG, et al. The bidirectional interactions between psoriasis and obstructive sleep apnea. Int J Dermatol 2015; 54: 1352-8.

8. Gupta MA, Simpson FC, Gupta AK. Bidirectional relationship between obstructive sleep apnea (OSA) and psoriasis: implications for OSA therapies? J Clin Sleep Med 2016; 12: 1309.

9. Cohen JM, Jackson CL, Li TY, et al. Sleep disordered breathing and the risk of psoriasis among US women. Arch Dermatol Res 2015; 307: 433-8.

10. Dalamaga M, Papadavid E, Vlami K. Unmasking the Janus face of the association between psoriasis, metabolic syndrome and obstructive sleep apnea. Sleep Breath 2013; 17: 449-50.

11. Egeberg A, Khalid U, Gislason GH, et al. Psoriasis and sleep apnea: a Danish Nationwide Cohort Study. J Clin Sleep Med 2016; 12: 663-71.

12. Shalom G, Dreiher J, Cohen A. Psoriasis and obstructive sleep apnea. Int J Dermatol 2016; 55: e579-84.

13. Gabryelska A, Sochal M, Wasik B, et al. Patients with obstructive sleep apnea are over four times more likely to suffer from psoriasis than the general population. J Clin Sleep Med 2018; 14: 153.
14. Ardic S, Demir AU, Ucar ZZ, et al. Prevalence and associated factors of sleep-disordered breathing in the Turkish adult population. Sleep Biol Rhythms 2013; 11: 29-39.

15. Ilbay VK, Tas B, Altuntas M, et al. Risk of obstructive sleep apnea syndrome in psoriasis patients. Arch Iran Med 2019; 22: 137-43.

16. Gisondi P, Rossini M, Di Cesare A, et al. Vitamin D status in patients with chronic plaque psoriasis. Br I Dermatol 2012; 166 505-10.

17. Ricceri F, Pescitelli L, Tripo L, Prignano F. Deficiency of serum concentration of 25 -hydroxyvitamin D correlates with severity of disease in chronic plaque psoriasis. I Am Acad Dermatol 2013; 68: 511-2.

18. Mason A, Mason J, Cork M, et al. Topical treatments for chronic plaque psoriasis: an abridged Cochrane systematic review. J Am Acad Dermatol 2013; 69: 799-807.

19. Scott LJ, Dunn CJ, Goa KL. Calcipotriol ointment. A review of its use in the management of psoriasis. Am J Clin Dermatol 2001; 2: 95-120.

20. Mangelsdorf DJ, Thummel C, Beato M, et al. The nuclear receptor superfamily: the second decade. Cell 1995; 83: 835-9.

21. Lee YH, Song GG. Association between circulating 25-hydroxyvitamin D levels and psoriasis, and correlation with disease severity: a meta-analysis. Clin Exp Dermatol 2018; 43: 529-35.

22. Amon U, Baier L, Yaguboglu R, et al. Serum 25-hydroxyvitamin $D$ levels in patients with skin diseases including psoriasis, infections, and atopic dermatitis. Dermatoendocrinology 2018; 10: e1442159.

23. Filoni A, Vestita M, Congedo M, et al. Association between psoriasis and vitamin D: duration of disease correlates with decreased vitamin D serum levels: an observational case-control study. Medicine 2018; 97: e11185.

24. Al-Dhubaibi MS. Association between Vitamin D deficiency and psoriasis: an exploratory study. Int J Health Sci (Qassim) 2018; 12: 33-9.

25. Ger TY, Fu Y, Chi CC. Bidirectional association between psoriasis and obstructive sleep apnea: a systematic review and meta-analysis. Sci Rep 2020; 10: 5931.

26. McCarty DE, Chesson Jr AL, Jain SK, Marino AA. The link between vitamin D metabolism and sleep medicine. Sleep Med Rev 2014: 311-9.

27. Piovezan RD, Hirotsu C, Feres MC, et al. Obstructive sleep apnea and objective short sleep duration are independently associated with the risk of serum vitamin D deficiency. PLoS One 2017; 12: e0180901.

28. Mete T, Yalcin Y, Berker D, et al. Obstructive sleep apnea syndrome and its association with vitamin $D$ deficiency. J Endocrinol Invest 2013; 36: 681-5.

29. Barcelo A, Esquinas C, Pierola J, et al. Vitamin D status and parathyroid hormone levels in patients with obstructive sleep apnea. Respiration 2013; 86: 295-301.

30. Erden ES, Genc S, Motor S, et al. Investigation of serum bisphenol A, vitamin D, and parathyroid hormone levels in patients with obstructive sleep apnea syndrome. Endocrine 2014; 45: 311-8.

31. Papadavid E, Dalamaga M, Vlami K, et al. Psoriasis is associated with risk of obstructive sleep apnea independently from metabolic parameters and other comorbidities: a large hospital-based case-control study. Sleep Breath 2017; 21: 949-58.

32. Ragia G, Archontogeorgis K, Simmaco M, et al. Genetics of obstructive sleep apnea: vitamin $\mathrm{D}$ receptor gene variation 
affects both vitamin D serum concentration and disease susceptibility. OMICS 2019; 23: 45-53.

33. Kirac D, Yagcioglu Yassa O, et al. Different VDR, VDBP genotypes and vitamin D levels may effect obstructive sleep apnea syndrome. Cell Mol Biol 2019; 65: 46-51.

34. Lee DY, Park BS, Choi KH, et al. Vitamin D receptor genotypes are not associated with clinical response to calcipotriol in Korean psoriasis patients. Arch Dermatol Res 2002; 294: 1-5.

35. Park BS, Park JS, Lee DY, et al. Vitamin D receptor polymorphism is associated with psoriasis. J Invest Dermatol 1999; 112: 113-6.

36. Liu J, Zeng HM, Lin MG, et al. Association of vitamin D receptor polymorphisms with susceptibility to psoriasis vulgaris and clinical response to calcipotriol in patients with psoriasis vulgaris. Chin J Dermatol 2017; 50: 889-93.

37. Dayangac-Erden D, Karaduman A, Erdem-Yurter H. Polymorphisms of vitamin $D$ receptor gene in Turkish familial psoriasis patients. Arch Dermatol Res 2007; 299: 487-91.

38. Kaya TI, Erdal ME, Tursen U, et al. Association between vitamin $\mathrm{D}$ receptor gene polymorphism and psoriasis among the Turkish population. Arch Dermatol Res 2002; 294: 286-9.

39. Okita H, Ohtsuka T, Yamakage A, Yamazaki S. Polymorphism of the vitamin $D(3)$ receptor in patients with psoriasis. Arch Dermatol Res 2002; 294: 159-62.

40. Richetta AG, Silvestri V, Giancristoforo S, et al. A-1012G promoter polymorphism of vitamin $\mathrm{D}$ receptor gene is associated with psoriasis risk and lower allele-specific expression. DNA Cell Biol 2014; 33: 102-9.

41. Rucevic I, Stefanic M, Tokic S, et al. Lack of association of vitamin D receptor gene 3'-haplotypes with psoriasis in Croatian patients. J Dermatol 2012; 39: 58-62.

42. Zuel-Fakkar NM, Kamel MM, Asaad MK, et al. A study of Apal and Taql genotypes of the vitamin D receptor in Egyptian patients with psoriasis. Clin Exp Dermatol 2011; 36: 355-9.

43. Li J, Sun L, Sun J, Yan M. Pooling analysis regarding the impact of human vitamin $D$ receptor variants on the odds of psoriasis. BMC Med Genet 2019; 20: 161. 